Research Article

\title{
Identification of Key Influencing Factors of User Experience of Mobile Reading APP in China Based on the Fuzzy-DEMATEL Model
}

\author{
Yi Zhang $(\mathbb{D}$, Xing Rong $(\mathbb{D}$, Mengfei Shu $\mathbb{D}$, and Qiang Chen $(\mathbb{D})$ \\ School of Economics and Management, Hubei University of Technology, Wuhan 430068, Hubei, China \\ Correspondence should be addressed to Yi Zhang; 20130031@hbut.edu.cn
}

Received 10 April 2021; Revised 15 May 2021; Accepted 21 May 2021; Published 11 June 2021

Academic Editor: Ali Ahmadian

Copyright (c) 2021 Yi Zhang et al. This is an open access article distributed under the Creative Commons Attribution License, which permits unrestricted use, distribution, and reproduction in any medium, provided the original work is properly cited.

\begin{abstract}
With the rapid development of the mobile Internet, mobile reading has transcended the limitation of time and space, becoming an indispensable part of people's living and learning. As the direct service object of mobile reading APP, users' subjective use experience is decisive in the success or failure. It is of great significance to identify the key influencing factors of user experience of mobile reading APP. It is conducive not only to improving users reading experience and increasing users stickiness but also to improving mobile reading service quality, which is essential to promote the healthy and sustainable development of mobile reading APP. From the perspective of user experience, content analysis and questionnaire survey are used to construct the index system of influencing factors of mobile reading APP user experience, which includes 6 dimensions of reliability, usefulness, thoughtfulness, interactivity, security, and compatibility, and 21 influencing factors. Based on the decision-making trial and evaluation laboratory (DEMATEL) method, introducing the triangular fuzzy number, the fuzzy decision-making trial and evaluation laboratory (fuzzy-DEMATEL) model is established, extending the single value of the comparison matrix to a fuzzy interval and then giving the decision makers a proper judgment space. The key influencing factors of mobile reading APP user experience are identified by algorithm design. Ultimately, the 10 key influencing factors of mobile reading APP user experience are identified, which are information quality, content richness, value fit, copyright protection, timeliness, personalized service, reading function richness, interpersonal interaction, system performance, and privacy protection, and suggestions for improving the user experience of mobile reading APP are put forward to provide reference for mobile reading APP developers. The research results not only enrich and perfect the theoretical and practical research in the mobile reading field to a certain extent, providing a new research perspective for the field of mobile reading, but also effectively solve the defects of the traditional DEMATEL method that expert judgment is subjectively biased and difficult to express with an exact number directly. The research expands the application scope of DEMATEL.
\end{abstract}

\section{Introduction}

With the rapid development of mobile Internet and the wide use of mobile terminal represented by smart phones and tablet computers, mobile reading has become an important way for people to acquire knowledge. People's reading time is becoming more and more fragmented, and mobile reading has become an indispensable part of people's living and learning due to the unrestricted time and space of mobile reading. Mobile reading refers to a new way of reading which uses mobile reading devices as the carrier to download or receive text information, images, sounds, data, and other forms of information published and distributed on the Internet in the electronic form via a portable reading terminal through wire or wireless. According to the "2020 China Mobile Reading Industry Development Special Research Report" released by iiMedia and the "2020 China Mobile Reading Industry Report" released by Mob Research Institute, the scale of China's mobile reading market has reached 37.21 billion yuan [1], the scale of China's online reading users has reached 530 million [1], and the average monthly usage time has reached 16 hours [2]. The mobile 
reading industry continued to develop steadily [3]. According to the unlimited expansion of the capacity of electronic products, simple and fast search, situational realtime recommendation, and other functions, mobile reading is favored by consumers and occupies the reading market rapidly and plays an important role in the steady progress of social reading [4]. The mobile reading industry in China has a broad prospect at present, but there are some disadvantages in the mobile reading APP, such as content homogeneity, incomprehensive service, low security, and so on, which lead to poor user experience and insufficient user stickiness. User experience is the key to the long-term development of mobile reading APP developers. Therefore, identifying the key influencing factors of user experience is of great significance to improve user reading experience, promote the quality of mobile reading service, and enhance the healthy and sustainable development of mobile reading APP.

In the research results related to the mobile reading, scholars mainly focused on mobile reading terminal software, user groups, reading operation mode, and reading service [5]. A lot of scholars also studied the development trend, development path, and development strategy of mobile reading APP [6-9]. Some scholars further explored the continuous use behavior [10-12], knowledge sharing behavior [13], payment behavior [14], withdrawal behavior [15], reading literacy of mobile reading users [16], and so on. Moreover, some scholars studied mobile reading user satisfaction [17-19], mobile reading service quality [20], and mobile reading APP availability [21]. However, research on mobile reading user experience is rare $[22,23]$. In the research results related to the identification method of key influencing factors, most scholars used the DEMATEL model [24-28], while some scholars used data statistical analysis methods, such as the structural equation model [29], complex social network analysis method [30], and Pearson correlation coefficient analysis method [31]. Some experts and scholars integrated two or more mathematical models on the basis of previous studies, such as the DEMATEL-ISM model [32], grey-DEMATEL model [33], AHP-DEMATEL model [34], and BP-DEMATEL model [35], which have enriched the theoretical and practical research on the identification method of key influencing factors to a certain extent.

Although the research results above have solved some problems, the following aspects still need to be improved. (1) Most of the existing studies analyze the behavior characteristics of mobile readers, but the application and research of user experience in the field of mobile reading is rare, and there is little literature to explore the user experience of mobile reading in-depth. (2) The existing research is mainly aimed at measuring the usability of mobile reading APP at a certain level, which is one-sided and lacks a systematic and comprehensive analysis of the key influencing factors of mobile reading APP user experience. (3) The traditional DEMATEL method requires the full use of expert experience and knowledge to judge the affecting degree between each two factors, but there remains uncertainty in their judgment, which is difficult to express directly with an accurate number; therefore, a more rigorous method of causal inference is required. From the perspective of user experience, this study uses content analysis and questionnaire survey to construct the index system of influencing factors of mobile reading APP user experience. Based on the DEMATEL method, introducing the triangular fuzzy number, the fuzzyDEMATEL model is established, extending the single value of the comparison matrix to a fuzzy interval and then giving the decision makers a proper judgment space. The key influencing factors of mobile reading APP user experience are identified by algorithm design, and suggestions for improving the user experience of mobile reading APP are put forward to provide reference for mobile reading APP developers. This study not only enriches and perfects the theoretical and practical research in the field of mobile reading to a certain extent, providing a new research perspective for the field of mobile reading, but also effectively solve the defects of the traditional DEMATEL method that expert judgment is subjectively biased and difficult to express with an exact number directly. The research expands the application scope of DEMATEL.

The following section is construction of the index system of influencing factors of mobile reading APP user experience. Section 3 is methodology. Section 4 is results and discussion. Finally, conclusions and suggestion of this study is drawn.

\section{Construction of the Index System of Influencing Factors of Mobile Reading APP User Experience}

2.1. Index System Framework of Influencing Factors of User Experience. Mobile reading APP user experience research studies have a late start, and there are less research results. Mobile reading APP is a product, and it has all the attributes of a product. This study constructs the index system framework of influencing factors on user experience of mobile reading APP by analyzing and summarizing relevant research studies on product user experience as references. This study refers to the $5 \mathrm{E}$ model (including effective, efficient, engaging, error tolerant, and easy to learn) proposed by Quesenbery [36], the honeycomb model (including usable, useful, accessible, credible, findable, desirable, and valuable) proposed by Morville [37], and the APEC model (including aesthetic, practical, emotional, and cognitive) proposed by Vyas and Gerrit [38]. Combining the application environment and characteristics of mobile reading APP, this study constructs the index system framework of influencing factors of user experience in mobile reading APP from six dimensions: reliability, usefulness, thoughtfulness, interactivity, security, and compatibility.

\subsection{Construction of the Index System of Influencing Factors.} Based on the guiding principle, systematic principle, scientific principle, and operational principle, this study uses content analysis and questionnaire survey to construct the index system of influencing factors of mobile reading APP user experience. First of all, the content analysis method was 
used to identify, encode, and summarize the indexes from the relevant literature. "Taking "mobile reading," "digital reading," mobile APP," "user experience," "user satisfaction," "user perception," "willingness to use," influencing factors," "measurement," and "evaluation" as search terms, the retrieval time was from 1980 to 2020. After selection, 28 influencing factors of mobile reading APP user experience were obtained. Then, the index system was revised by user questionnaire. The respondents, aged 13-50, were students and young and middle-aged workers. A total of 500 questionnaires were distributed, 462 questionnaires were collected, accounting for $92.4 \%$, among which 430 are valid questionnaires, accounting for $86 \%$. An index system of influencing factors of user experience of mobile reading APP are preliminary identified which includes 6 dimensions of reliability, usefulness, thoughtfulness, interactivity, security, and compatibility and 21 influencing factors and the corresponding items are given in Table 1.

\subsection{Explanation of Influencing Factors}

2.3.1. Reliability. Reliability refers to the ability of mobile reading software to perform its service reliably and accurately, which mainly includes four factors: fluency, availability, system performance, and stability. Fluency refers to when the users are in the use of mobile reading software, and the software system has short response time and run fast without time lag, saving users' time. Availability refers to obtain the required information and experience from free resources and service and provide cost-effective and free service. System performance refers to the high equipment system compatibility, high configuration, small software installation package, and be easy for users to use equipment memory efficiently. Stability refers to the less accidents in the process of use in order to ensure the normal operation of the software, which can be shown as the less flashback, low error rate, timely access to specific usage scenarios, finding problems, repair vulnerabilities, and low incidence of memory crashes, creating a reliable and stable use environment for users.

2.3.2. Usefulness. Usefulness refers to the degree that the reading resources provided by mobile reading software can meet the reading needs of users, including four factors: content richness, information quality, timeliness, and integrity. Content richness refers to the amount and variety of reading resources provided by mobile reading software. Information quality means that mobile reading software can screen reading resources to ensure books or articles are of high quality, and original articles are innovative which promotes users' trust in reading software. Timeliness refers to the ability to effectively push the latest hot reading resources to users, that is, mobile reading software can provide articles that follow the real-time hot spots to ensure a high rate of book replacement and update rate of mobile reading information resources. Integrity means that mobile reading software can provide complete services covering personal center, reading, learning, socializing, book shopping, and other fields, which can meet the needs of users in many aspects.

2.3.3. Thoughtfulness. Thoughtfulness refers to the degree of thoughtfulness that the service mobile reading software can provide, which mainly includes four factors: personalized service, convenience, rich reading function, and advertising. Personalized service refers to automatic classification of what users have read and content pushing according to the users' preference. The personal center setting is novel, such as reminder, inventory, and other service functions that can be set according to the users' expectations, satisfying users' personal preferences. Convenience means that users can easily and quickly retrieve and obtain information when using reading software and can search for articles or books that have been browsed a long time ago, facilitating users to access the content they have read before. The software can provide reminders to facilitate users to clean up buffer files in time. Rich reading function means that mobile reading software can provide directory and bookmark function, night mode, brightness adjustment, magnifying glass, reading interface adapting to mobile phone interface, set font size, page turning mode simulation, and other functions to improve the users' real-time reading experience. Providing a variety of note-taking forms, users can quickly mark, query, and share one-click pictures or links, enhancing the users' stickiness and recognition for the software. Advertising refers to less advertisement pushing in reading page, no malicious advertising, and no bundled software, which can reduce the interference in the process of reading.

2.3.4. Interactivity. Interactivity refers to the degree of interaction between users and mobile reading software as well as other users in different ways in the process of using mobile reading software, including three factors such as interface design, interpersonal interaction, and feedback system. Interface design refers to the beauty and comfort level of the software interface. First, the layout of content are reasonable which is logical in operation; the common functions such as "search," "rank," and so on are placed in the conspicuous position which leads to a good browsing effect. Second, icon design is full of personality, and typography is of strong readability. Third, the color collocation of interface is coordinate and has a strong sense of esthetics. Interface is the medium to convey information between users and software, and its design will greatly affect the users' willingness to use. Interpersonal interaction refers to the communication degree of users through mobile reading platforms. First, software provides community service functions and supports the initiation of topics or activities and classifies them, which can create opportunities for users to get acquainted and communicate with others of the same interests. Second, as for community sharing, users can carry out basic social interaction behaviors such as forwarding, commenting, and rewarding. Third, with interactive functions of following and chatting, users' demand of making friends is fully satisfied. Fourth, community has a certain supervision and 
TABLE 1: Index system of influencing factors of user experience of mobile reading APP.

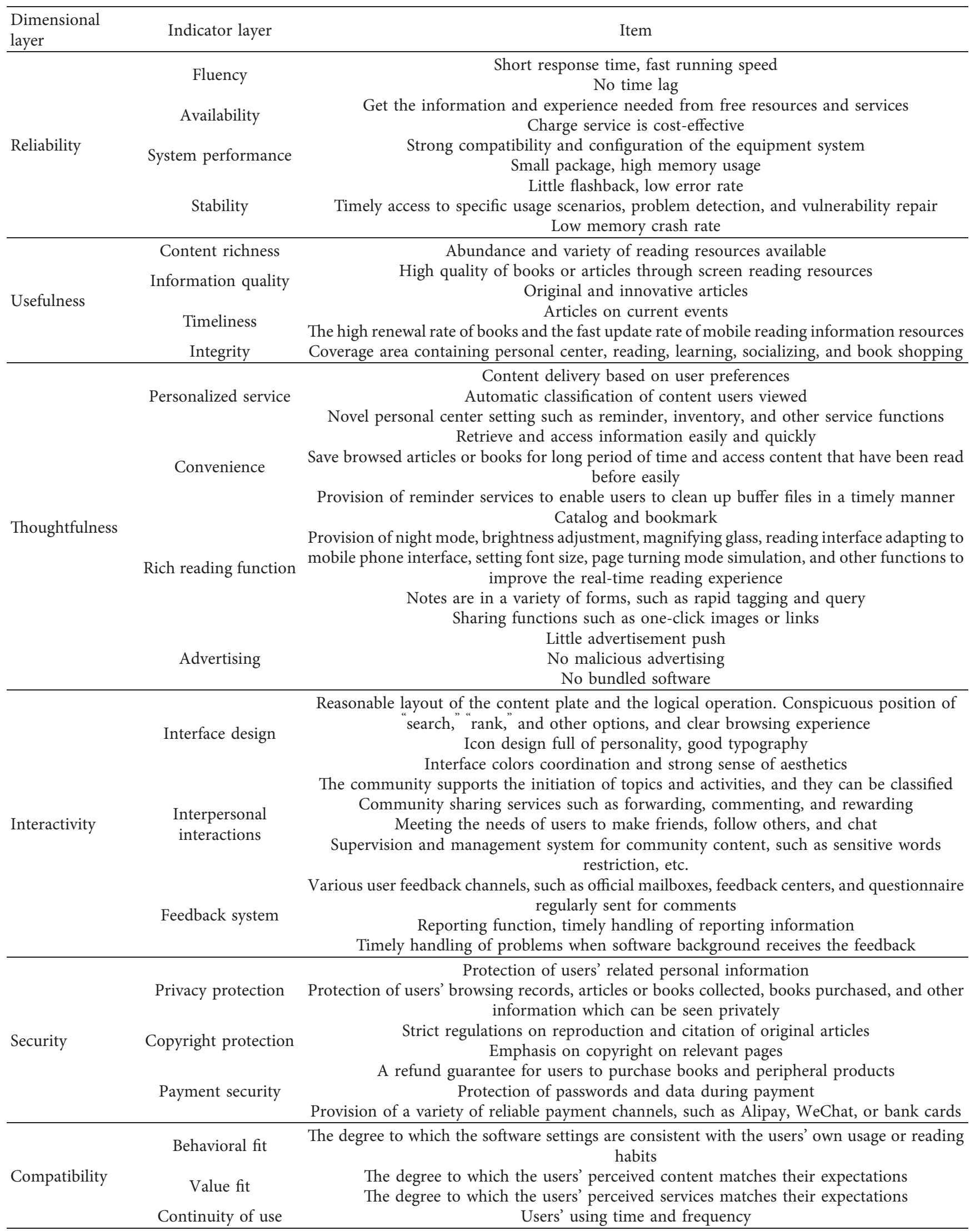


management system for community content, and there are sensitive word restrictions, so as to avoid adverse content affecting users' reading experience. Feedback system refers to a way of transmitting information and communicating between users and customer-service staffs which reflected in variety of users' feedback channels that the software can provide. For instance, the software can provide official mailbox, establish feedback center, regularly ask users for comments, provide reporting function, timely identify and handle reported information, deal with feedbacks timely at the backstage, and so on. The feedback mechanism can effectively promote communication between users and software and enhance user experience.

2.3.5. Security. Security refers to the degree to which the users' privacy and information are protected when using mobile reading software, including three factors such as privacy protection, copyright protection, and payment security. Privacy protection refers to the degree of software protection of user-related information, such as users' personal information, browsing records, articles or books collected, books purchased, and other information. Copyright protection means that the software has strict control over the reprint and citation for the users' original articles, emphasizes the copyright in relevant pages, embodying a high degree of copyright protecting consciousness, and can protect the intellectual property rights of the users. Payment security refers to the security of payment function in mobile reading software. For example, the software can provide a refund guarantee when users purchase books and peripheral products, protect the password and data during payment, and provide a variety of reliable payment channels, such as Alipay, WeChat, or bank card, to ensure the security of user payment environment.

2.3.6. Compatibility. Compatibility refers to the degree to which the value perceived by users after using the software conforms to the value that users expect, which mainly includes three factors such as behavioral fit, value fit, and continuity of use. Behavioral fit refers to the degree of conformity between the software settings and the users' habits of using or reading. Value fit refers to the degree of conformity between the content and service perceived and expected by users. The higher the behavioral fit and value fit, the more appropriate to users' initial expectations of the software. Continuity of use refers to the length of users' use time and frequency, which is the embodiment of users' specific behavior. The longer using time and the more times of use indicate the higher degree of users' dependence and recognition of the software.

\section{Methodology}

3.1. Selection of Research Methods. DEMATEL is a kind of analytical method based on graph theory and matrix, which is used to screen the main influencing factors of the complex system and simplify the system structure. It is widely used in knowledge discovery, information resource sharing, and so on. At present, DEMATEL has developed into a mature theoretical method system, but the application of this method still has some limitations. The method needs to make full use of the expert experience and knowledge to judge the affecting degree between each two factors, but there remains uncertainty in expert judgment in most cases, so it is difficult to express judgment directly with a precise number. Fuzzy-DEMATEL is a factor analysis method based on DEMATEL with the introduction of fuzzy mathematics [39-45]. By introducing the triangular fuzzy number method, the single value of the comparison matrix can be extended to a fuzzy interval to give the decision maker a proper judgment space. The fuzzy-DEMATEL model can effectively solve the defects of the traditional DEMATEL method.

3.2. Fuzzy-DEMATEL Model. The initial direct-relation matrix is constructed according to the correspondence between the expert semantic code and the triangular fuzzy number given by Li [46]. On this basis, the converting fuzzy data into crisp scores (CFCS) method is used to clarify the initial direct-relation matrix to obtain the direct-relation matrix as the initial matrix of the DEMATEL method, and then, key influencing factors are identified by the DEMATEL method. The specific steps of fuzzy-DEMATEL algorithm are given in Algorithm 1.

\section{Step 1. Set up judgment matrix}

The influencing factors are $a_{1}, a_{2}, \cdots, a_{n}$. Set up the judgment matrix $\mathrm{A}$ through the questionnaire about the experts' judgment of affecting degree between each two factors. In this matrix, the element $a_{i j}$ represents the affecting degree of factor $a_{i}$ on factor $a_{j}$. The affecting degree is divided into five grades from weak to strong as no influence, weak influence, certain influence, strong influence, and extremely strong influence, respectively, and corresponds to $0,1,2,3$, and 4 .

\section{Step 2. Set up initial direct-relation matrix}

Process the judgment matrix $A$ using triangular fuzzy number and obtain the initial direct-relation matrix $B$. The element $a_{i j}$ in the judgment matrix $A$ is transformed into $\left(s_{i j}, m_{i j}, u_{i j}\right)$ according to the correspondence between expert semantic code and triangular fuzzy number as given in Table 2 . The $s_{i j}$ represents the minimum possible value of the affecting degree of the factor $a_{i}$ on the factor $a_{j}, m_{i j}$ represents the most probable value of the affecting degree of the factor $a_{i}$ on the factor $a_{j}$, and $u_{i j}$ represents the maximum possible value of the affecting degree of the factor $a_{i}$ on the factor $a_{j}$.

Step 3. Construct direct-relation matrix

The triangular fuzzy number obtained from the expert semantic evaluation cannot be directly used for data analysis and processing, so it is necessary to do the defuzzification of triangular fuzzy numbers to obtain accurate values. This study uses the CFCS method to clarify the matrix $\mathrm{B}$ and obtain the direct-relation 
TABle 2: Correspondence between expert semantic code and triangular fuzzy number.

\begin{tabular}{lc}
\hline Expert evaluation semantic code & Triangular fuzzy number \\
\hline No influence & $(0,0,0.25)$ \\
Weak influence & $(0,0.25,0.5)$ \\
Certain influence & $(0.25,0.5,0.75)$ \\
Strong influence & $(0.5,0.75,1)$ \\
Extremely strong influence & $(0.75,1,1)$ \\
\hline
\end{tabular}

matrix $Z$, so that the transformation loss of the numerical value is relatively small. Suppose a total of $Y$ experts participate in the evaluation of the affecting degree between each two factors, the triangular fuzzy number corresponding to the affecting degree of factor $a_{i}$ on factor $a_{j}$ evaluated by expert $k$ is denoted by $z_{i j}^{k}=\left(s_{i j}^{k}, m_{i j}^{k}, u_{i j}^{k}\right), \quad(k=1,2, \cdots, Y)$. The steps of the CFCS method are as follows:

(1) Standardize the triangular fuzzy number

$$
\begin{gathered}
x s_{i j}^{k}=\frac{s_{i j}^{k}-\min s_{i j}^{k}}{\Delta_{\min }^{\max }} ; \\
x m_{i j}^{k}=\frac{m_{i j}^{k}-\min m_{i j}^{k}}{\Delta_{\min }^{\max }} ; \\
x u_{i j}^{k}=\frac{u_{i j}^{k}-\min u_{i j}^{k}}{\Delta_{\min }^{\max }}
\end{gathered}
$$

Among them, $\Delta_{\min }^{\max }=\max u_{i j}^{k}-\min s_{i j}^{k}$

(2) Standardize the left value and right value of the triangular fuzzy number

$$
\begin{aligned}
x s s_{i j}^{k} & =\frac{x m_{i j}^{k}}{1+x m_{i j}^{k}-x s_{i j}^{k}} ; \\
x u s_{i j}^{k} & =\frac{x u_{i j}^{k}}{1+x u_{i j}^{k}-x m_{i j}^{k}} .
\end{aligned}
$$

(3) Calculate the clear value of each expert evaluation's fuzzy value after defuzzification

$z_{i j}^{k}=\min s_{i j}^{Y}+\left[\frac{x s s_{i j}^{k}\left(1-x s s_{i j}^{k}\right)+\left(x u s_{i j}^{k}\right)^{2}}{1-x s s_{i j}^{k}+x u s_{i j}^{k}}\right] \Delta_{\min }^{\max }$.

(4) Calculate the average clear value and get directrelation matrix $Z$

$$
\begin{aligned}
z_{i j}= & \frac{1}{Y}\left(z_{i j}^{1}+z_{i j}^{2}+, \cdots,+z_{i j}^{k}\right), \\
Z=\left(z_{i j}\right)_{n \times n}= & {\left[\begin{array}{cccc}
0 & z_{12} & \cdots & z_{1 n} \\
z_{21} & 0 & \cdots & z_{2 n} \\
\vdots & \vdots & \ddots & \vdots \\
z_{n 1} & z_{n 2} & \cdots & 0
\end{array}\right] . }
\end{aligned}
$$

Step 4. Set up the generalized direct-relation matrix

Taking the direct-relation matrix $Z$ as a molecule and the maximum of the sum of rows and the sum of columns as denominator to do linear operations on the matrix, then the generalized direct-relation matrix $D$ is obtained.

$$
D=\frac{Z}{\max \left\{1 \leq i \leq n^{\max } \sum_{j=1}^{n} z_{i j} \max _{1 \leq i \leq n} \sum_{i=1}^{n} z_{i j}\right\}} .
$$

Step 5. Set up the total-relation matrix

The generalized direct-relation matrix is a binary-relation matrix, which can only describe the direct effects between factors, while binary or multivariate effects occur simultaneously in a complex system. Therefore, the total-relation matrix $T$ needs to be calculated.

$$
T=D^{1}+D^{2}+D^{3}+, \cdots,+D^{n} .
$$

As $n$ approaches infinity, $T=D\left(I-D^{-1}\right)$

Among them, $I$ is a unit matrix

Step 6. Calculate the influence degree and influenced degree of each factor

The influence degree $R_{i}$ and influenced degree $C_{i}$ of the factor $i$ are calculated according to the total-relation matrix $T$. The influence degree $R_{i}$ of the factor $i$ is the sum of the $n$ elements in row $i$ of the total-relation matrix $T$, and the influenced degree $C_{i}$ of the factor $i$ is the sum of the $n$ elements in column $i$ of the totalrelation matrix $T$.

$$
\begin{aligned}
R_{i} & =\sum_{j=1}^{n} t_{i j}, \\
C_{i} & =\sum_{i=1}^{n} t_{i j} .
\end{aligned}
$$

Step 7. Calculate the center degree and cause degree of each factor

The center degree $E_{i}$ and cause degree $F_{i}$ of the factor $i$ are calculated according to the influence degree $R_{i}$ and influenced degree $C_{i}$ of the factor $i$.

$$
\begin{aligned}
& E_{i}=R_{i}+C_{i}, \\
& F_{i}=R_{i}-C_{i} .
\end{aligned}
$$

The greater the value of center degree $E_{i}$, the greater the importance of the factor in the system. If the cause degree $F_{i}>0$, it is the cause factor; if the cause degree $F_{i}<0$, it is the result factor.

\section{Step 8 . Construct a causality graph}

Taking the center degree of each factor as the horizontal coordinate and the cause degree of each factor as the vertical coordinate, the causality diagram is constructed. The points with vertical coordinate above 0 are cause factors, the points with vertical coordinate below 0 are result factors, and the factor from left to 
right is increasingly more important in the system. The key factors of the system are identified according to the center degree of each factor.

\section{Results and Discussion}

4.1. Data Acquisition and Analysis. Invite 21 experts to evaluate affecting degree between each two factors according to the five-level $(0,1,2,3,4)$ scoring rules. By using Matlab 2018a to program and calculate the center degree and cause degree of each factor, the concrete result obtained is given in Table 3.

According to the data in Table 3, the causality diagram is drawn with the center degree of each factor as the horizontal coordinate and the cause degree of each factor as the vertical coordinate, as shown in Figure 1.

Figure 1 shows the causality diagram of 21 influencing factors of mobile reading APP user experience. The horizontal coordinate represents the center degree and the vertical coordinate represents the cause degree. In terms of cause degree, points with vertical coordinate above 0 are cause factors, and the sequence numbers of the 5 factors with the largest cause degree (cause degree $>0.5$ ) are 5 (content richness), 20 (value fit), 17 (copyright protection), 9 (personalized service), and 3 (system performance). The points with vertical coordinate below 0 are the result factors. The sequence numbers of the 4 factors with the largest absolute value of cause degree (cause degree $<-0.5$ ) are 6 (information quality), 7 (timeliness), 11 (rich reading function), and 14 (interpersonal interactions). In terms of center degree, the sequence numbers of the 10 factors with the largest center degree (center degree $>2.8$ ) are 6 (information quality), 5 (content richness), 20 (value fit), 17 (copyright protection), 7 (timeliness), 9 (personalized service), 11 (rich reading function), 14 (interpersonal interactions), 3 (system performance), and 16 (privacy protection).

\subsection{Results Analysis and Discussion}

4.2.1. Identification of Cause and Result Factors. It can be found from Table 3 and Figure 1 that 10 of the 21 influencing factors are cause factors (cause degree $>0$ ). From the largest cause degree to the smallest, the cause factors are content richness, value fit, copyright protection, personalized service, system performance, convenience, behavior fit, availability, stability, and interface design. It shows that these factors are more inclined to affect other factors. Among them, the 5 factors with the largest cause degree (cause degree $>0.5$ ) are content richness, value fit, copyright protection, personalized service, and system performance. The details are given in Table 4.

Through Table 3 and Figure 1, it can be observed that 11 out of 21 influencing factors are result factors (cause degree $<0$ ). From the largest absolute value of cause degree to the smallest, the result factors are information quality, timeliness, rich reading function, interpersonal interactions, privacy protection, payment security, feedback system, integrity, advertising, fluency, and continuity of use. It shows that they are more influenced by other factors. Among them, the 4 factors with the largest absolute value of cause degree (cause degree $<-0.5$ ) are information quality, timeliness, rich reading function, and interpersonal interactions. The details are given in Table 5 .

4.2.2. Identification of Key Influencing Factors. The center degree is regarded as the basis for judging the importance of factors. According to Table 3, the factors are sorted from large to small by center degrees, and the results are given in Table 6.10 factors with the center degree higher than the average value of 2.8 are selected as the key influencing factors, which are information quality, content richness, value fit, copyright protection, timeliness, personalized service, rich reading function, interpersonal interactions, system performance, and privacy protection.

4.2.3. Fuzzy-DEMATEL Methodology Discussion. General DEMATEL methods usually require accurate and specific numerical value to define the affecting degree between each two factors. However, the influencing factors of many practical problems often possess complexity and fuzziness, especially the research problems related to user experience. Experts' identification and judgment of influencing factors through the DEMATEL method will also have certain subjective bias due to individual differences in professional knowledge, research experience, and so on, thus affecting the accuracy of data analysis results of DEMATEL to a certain extent. Based on the DEMATEL method, introducing the triangular fuzzy number, the fuzzy-DEMATEL model is established, extending the single value of the comparison matrix to a fuzzy interval and then giving the decision makers a proper judgment space. The key influencing factors of mobile reading APP user experience are identified by algorithm design. It effectively solves the defects of the traditional DEMATEL method that expert judgment is subjectively biased and difficult to express with an exact number directly. The research expands the application scope of DEMATEL. 
TABLE 3: Center degree and cause degree of influencing factors of mobile reading APP user experience.

\begin{tabular}{lccc}
\hline Number of factors & Factors & Center degree & Cause degree \\
\hline 1 & Fluency & 2.76978 & -0.14739 \\
2 & Availability & 2.76731 & 0.08810 \\
3 & System performance & 2.81148 & 0.50902 \\
4 & Stability & 2.76706 & 0.07963 \\
5 & Content richness & 3.06627 & 1.33607 \\
6 & Information quality & 3.13706 & -1.49403 \\
7 & Timeliness & 2.91021 & -0.91378 \\
8 & Integrity & 2.77533 & -0.23046 \\
9 & Personalized service & 2.88299 & 0.82116 \\
10 & Convenience & 2.77723 & 0.25275 \\
11 & Rich reading function & 2.85406 & -0.71069 \\
12 & Advertising & 2.77223 & -0.18860 \\
13 & Interface design & 2.76637 & 0.04955 \\
14 & Interpersonal interactions & 2.84079 & -0.65425 \\
15 & Feedback system & 2.78690 & -0.34460 \\
16 & Privacy protection & 2.79646 & -0.41614 \\
17 & Copyright protection & 2.96525 & 1.07913 \\
18 & Payment security & 2.79201 & -0.38446 \\
19 & Behavioral fit & 2.77323 & 0.20300 \\
20 & Value fit & 3.01256 & 1.20525 \\
21 & Continuity of use & 2.76937 & -0.13925 \\
\hline
\end{tabular}

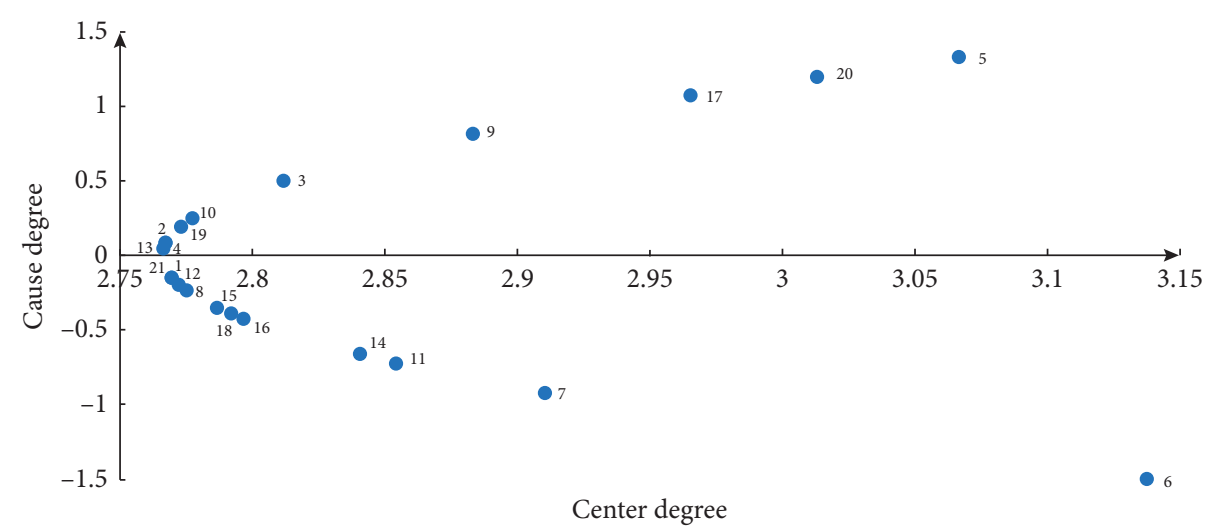

FIgURE 1: Causality diagram of influencing factors of mobile reading APP user experience.

The steps of the fuzzy-DEMATEL algorithm:

Step 1. Set up judgment matrix A through expert evaluation results;

Step 2. Process the judgment matrix A using triangular fuzzy number and obtain the initial direct-relation matrix B though correspondence between expert semantic code and triangular fuzzy number as given in Table 2;

Step 3. Defuzzify the initial direct-relation matrix B through the CSFC method to obtain direct-relation matrix Z:

Standardize the triangular fuzzy number;

Standardize the left value and right value of the triangular fuzzy number;

Calculate the clear value of each expert evaluation's fuzzy value after defuzzification;

Calculate the average clear value and get direct-relation matrix $Z$.

Step 4. Normalize the direct-relation matrix $Z$ to obtain the generalized direct-relation matrix $D$;

Step 5. Set up the total-relation matrix;

Step 6. Calculate the influence degree $R_{i}$ and influenced degree $C_{i}$ of each factor;

Step 7. Calculate the center degree $E_{i}\left(E_{i}=R_{i}+C_{i}\right)$ and cause degree $F_{i}\left(F_{i}=R_{i}-C_{i}\right)$ of each factor, and the results of center degree and cause degree are given in Table 3;

Step 8. Construct the causality diagram, taking the center degree of each factor as the horizontal coordinate and the cause degree of each factor as the vertical coordinate, as shown in Figure 1. 
TABle 4: Cause factors.

\begin{tabular}{lcc}
\hline Number of factors & Cause factors & Cause degree $(>0)$ \\
\hline 5 & Content richness & 1.33607 \\
20 & Value fit & 1.20525 \\
17 & Copyright protection & 1.07913 \\
9 & Personalized service & 0.82116 \\
3 & System performance & 0.50902 \\
10 & Convenience & 0.25275 \\
19 & Behavioral fit & 0.20300 \\
2 & Availability & 0.08810 \\
4 & Stability & 0.07963 \\
13 & Interface design & 0.04955 \\
\hline
\end{tabular}

TABLE 5: Result factors.

\begin{tabular}{lcc}
\hline Number of factors & Result factors & Cause degree $(<0)$ \\
\hline 6 & Information quality & -1.49403 \\
7 & Timeliness & -0.91378 \\
11 & Rich reading function & -0.71069 \\
14 & Interpersonal & -0.65425 \\
& interactions & -0.41614 \\
16 & Privacy protection & -0.38446 \\
15 & Payment security & -0.34460 \\
8 & Feedback system & -0.23046 \\
12 & Integrity & -0.18860 \\
1 & Advertising & -0.14739 \\
21 & Fluency & -0.13925 \\
\hline
\end{tabular}

TABLE 6: Center degree ranking of influencing factors of mobile reading APP user experience.

\begin{tabular}{lcc}
\hline Number of factors & Factors & Center degree \\
\hline 6 & Information quality & 3.13706 \\
5 & Content richness & 3.06627 \\
20 & Value fit & 3.01256 \\
17 & Copyright protection & 2.96525 \\
7 & Timeliness & 2.91021 \\
9 & Personalized service & 2.88299 \\
11 & Rich reading function & 2.85406 \\
14 & Interpersonal interactions & 2.84079 \\
3 & System performance & 2.81148 \\
16 & Privacy protection & 2.79646 \\
18 & Payment security & 2.79201 \\
15 & Feedback system & 2.78690 \\
10 & Convenience & 2.77723 \\
8 & Integrity & 2.77533 \\
19 & Behavioral fit & 2.77323 \\
12 & Advertising & 2.77223 \\
1 & Fluency & 2.76978 \\
21 & Continuity of use & 2.76937 \\
2 & Availability & 2.76731 \\
4 & Stability & 2.76706 \\
13 & Interface design & 2.76637 \\
& Mean value of center degree & 2.8 \\
\hline
\end{tabular}

\section{Conclusions and Suggestion}

\subsection{Conclusions}

\subsubsection{Theoretical Contribution}

(1) This study enriches and perfects the theoretical and practical research in the mobile reading field to a certain extent and provides a new research perspective for the field of mobile reading

Most of the existing studies analyze the behavior characteristics of mobile readers, but the application and research of user experience in the field of mobile reading is rare, and there is little literature to explore the user experience of mobile reading in-depth. The existing research is mainly aimed at measuring the usability of mobile reading APP at a certain level, which is one-sided and lacks a systematic and comprehensive analysis of the key influencing factors of mobile reading APP user experience. From the perspective of user experience, this study uses content analysis and questionnaire survey to preliminarily construct the index system of influencing factors of mobile reading APP user experience. On this basis, the fuzzy-DEMATEL model is established to identify the key influencing factors of mobile reading APP user experience. Ultimately, the $10 \mathrm{key}$ influencing factors of mobile reading APP user experience are identified, which are information quality, content richness, value fit, copyright protection, timeliness, personalized service, rich reading function, interpersonal interaction, system performance, and privacy protection, and suggestions for improving the user experience of mobile reading APP are put forward to provide reference for mobile reading APP developers. To some extent, this research result enriches and improves the theoretical and practical research in the mobile reading field and provides a new research perspective for the field of mobile reading.

(2) It effectively solves the defects of the traditional DEMATEL method that expert judgment is subjectively biased and difficult to express with an exact number directly. The research expands the application scope of DEMATEL.

The traditional DEMATEL method requires the full use of expert experience and knowledge to judge affecting degree between each two factors, but there remains uncertainty in their judgment, which is difficult to express directly with an accurate number; therefore, a more rigorous method of causal inference is required. Based on the DEMATEL method, introducing the triangular fuzzy number, the fuzzyDEMATEL model is established, extending the 
single value of the comparison matrix to a fuzzy interval and then giving the decision makers a proper judgment space, which provides a new method and idea for the identification of the key influencing factors of mobile reading APP user experience. The research results effectively solve the defects of the traditional DEMATEL method that expert judgment is subjective bias and difficult to express with an exact number directly and expand the application scope of DEMATEL.

5.1.2. Practical Contribution. With the rapid development of mobile Internet and the wide use of mobile terminal represented by smart phones and tablet computers, mobile reading has become an important way for people to acquire knowledge. People's reading time is becoming more and more fragmented, and mobile reading has become an indispensable part of people's living and learning due to the unrestricted time and space of mobile reading. The mobile reading industry in China has a broad prospect at present, but there are some disadvantages in the mobile reading APP, such as content homogeneity, incomprehensive service, low security, and so on, which lead to poor user experience and insufficient user stickiness. User experience is the key to the long-term development of mobile reading APP developers. Therefore, identifying the key influencing factors of user experience is of great significance to improve user reading experience, promote the quality of mobile reading service, and enhance the healthy and sustainable development of mobile reading APP.

5.2. Suggestion. On the basis of discussing the research results in the previous section, this study puts forward some suggestions on improving the user experience of mobile reading APP in theory and practice.

\subsubsection{Create High Quality Content, Control Screening} Process. For mobile platforms, the persistence of users using mobile reading software is greatly affected by the information quality, content richness, and value fit. On the basis of ensuring the amount and variety richness of reading resources, mobile reading software developers should first attach importance to the quality of information to meet the diverse needs of users and tolerate different values. However, it is of high cost in the early to achieve rich and comprehensive content coverage, and it is not suitable for products which are in the earlier market stage and with weak profitability, so this kind of platforms should improve user experience from two aspects: information quality and value fit. In terms of information quality, the platform needs to create high quality content. It is necessary for the software backstage to improve the content identification, screening, and updating mechanisms to ensure the high quality of books and articles placed on the platform, and this is a key to achieve user growth and improve user stickiness. For the mobile reading software focusing on news information, we should strike the balance between timeliness and information quality and strengthen examination and supervision on platform. In terms of value fit, because of products becoming more and more vertical, it is important to obtain the value recognition of users and make users take the platform as the first choice when they choose to read books. The platform can focus on some kinds of books to form a characteristic of unique content in the mind of users and become the preferred platform for users in related content fields. In addition, with the strengthening of people's copyright awareness, operators are expected to pay more attention to book authors and other article creators when expanding content scope in order to embody respect for creators' achievements in the platform.

\subsubsection{Provide Personalized Services to Meet Diverse Needs.} At present, the mobile reading platform is increasingly close to big data. When the platform creates a better service system by tracking user behavior, it also needs to pay attention to tracking the changing habits and needs of users with the changes of times. Mobile reading platforms with social and personalized service are more attractive than monotonous content aggregation. Mobile reading service platform should be innovative in reading function. For one thing, the platform should adhere to the user-oriented principle and aim at improving the users' reading experience, through enriching the platform's reading function. For another, keeping up with the development of science and technology, the platform should use artificial intelligence and big data to explore the needs of users, so as to categorize user groups, integrate the content effectively and push oriented contents, realizing the accurate matching of content and users, and giving users adequate alternatives. Among them, social function is an important channel to possess the information of users' behavior, which can improve the users' activity and capture users' operation track. Therefore, a deeper level of functional innovation requires mobile reading operators to constantly innovate and explore the interaction between users and platforms and users and users.

\subsubsection{Maintain Platform Stability and Ensure Privacy Se-} curity of Users. As online service software, the strong system performance and privacy protection are the important preconditions for the user to choose the platform. The mobile reading platform should pay attention to the system performance and ensure the security of users' personal information at the same time. First of all, the platform should strengthen the construction of the backstage security system, improve the stability of the platform, and ensure the security of users' personal information and operation track data. Additionally, it is of significance to strengthen the control of bad information and links in the process of platform operation, supervise, and prevent the abnormal user behavior in time. Finally, the platform should restrict its own behavior of reading or using user information at will, pay attention to ensure moderation when using big data and artificial intelligence technology to analyze user behavior, and avoid users falling into the dilemma of "information cocoons," so as to build a bridge of trust between users and 
platforms and create an atmosphere for users to feel at ease and read attentively.

\subsection{Limitations and Future Research}

(1) Key influencing factors of mobile reading APP user experience need further improvement

The key influencing factors identified in this study are scientific and systematic. Under the background of the rapid development of artificial intelligence, the indicators can be constantly improved and corrected on the basis of the index system of influencing factors, and more comprehensive and systematic key influencing factors can be identified in the future. Meanwhile, the number of samples in more regions and groups can be increased, leading to enhanced accuracy and applicability of key influencing factors.

(2) Future research on case analysis of mobile reading APP user experience

The case analysis of mobile reading APP such as WeChat reading, QQ reading, QiDian reading, and iReader can be conducted in the future research, so as to have a better understanding of the user experience of mobile reading APP and then improve user stickiness and gain long-term trust and support from users.

(3) Future research on group decision theory

As for the fuzzy-DEMATEL model established in this study, a number of experts participate in the judgment of affecting degree between each two factors. This study simply calculates the average clear value which is after the defuzzification of fuzzy value of each expert, but it lacks in-depth discussion on how to effectively integrate the expert opinions, leading to the information loss easily in average calculation, so it is necessary to deal with the expert opinions in a more rigorous way. In the actual evaluation work, a large number of expert evaluation and judgment results are the basis of the evaluation outcome. Therefore, how to effectively gather the evaluation opinions of multiple experts has become a core issue that needs to be handled, and the aggregation mode of multiexpert opinions needs further improvement. As a reasoning method of uncertainty, the core of D-S evidence theory is to provide a synthetic algorithm that can integrate multiple sources of data, which can effectively process uncertain information during the judgment process. Therefore, D-S evidence theory can be introduced to be combined with the fuzzy-DEMATEL model in the future research, so as to explore how to integrate expert opinions effectively.

\section{Data Availability}

The data used to support the findings of this study are available from the corresponding author upon request.

\section{Conflicts of Interest}

The authors declare that they have no conflicts of interest.

\section{Acknowledgments}

This work was supported by the National Natural Science Foundation of China (71904046).

\section{References}

[1] iiMedia, "Special research report on the development of China's mobile reading industry in 2020," iiMedia, Hong Kong, China, 2021, https://www.iimedia.cn/c400/77511.html.

[2] Mob Research Institute, "Report on China's mobile reading industry in 2020," Mob Research Institute, Shanghai, China, 2021, http://www.cet.com.cn/wzsy/cyzx/2763159.shtml.

[3] Analysys, Annual Comprehensive Analysis of China's Mobile Reading Market in 2020, Analysys, Beijing, China, 2020, https://www.analysys.cn/article/detail/20019686.

[4] B. Y. Wang, "Review on the progress of mobile reading service quality evaluation system," New Century Library, vol. 40, no. 1, pp. 86-91, 2019.

[5] K. Ma and X. L. Zhang, "Review on the research of mobile reading in China," Library, vol. 4, pp. 68-71, 2013.

[6] H. W. Xie, Y. H. Huo, and J. Nie, "Research on the development trend of mobile digital reading," Publishing Journal, vol. 21, no. 6, pp. 7-12, 2013.

[7] H. Y. A. Wang, "Study on the situation, problems and countermeasures of the mobile reading APPs," Researches in Library Science, vol. 37, no. 1, pp. 84-87, 2015.

[8] B. Li, Development Situation and Community Development Strategy of Mobile Reading APP, pp. 35-38, Modern Publishing, Delhi, India, 2016.

[9] S. Y. Feng, "The research on the development strategy of mobile reading APP-A case study of Net Ease Snail Reading," Researches in Library Science, vol. 23, pp. 85-89+14, 2018.

[10] L. Zeng, T. Cong, and Y. X. Zeng, "Research on mobile phone reading software continuous behavior," Publishing Journal, vol. 22, no. 1, pp. 84-88, 2014.

[11] D. G. Zhu, "Research on the influence of E-service quality on the continuance intention of social reading APPs," Journal of Modern Information, vol. 39, no. 4, pp. 76-85, 2019.

[12] W. J. Zhao and J. Y. Sun, "Research on college students' mobile reading behavior under the framework of information behavior: taking the superstar mobile reading APP platform as an example," Knowledge Management Forum, vol. 5, no. 1, pp. 10-23, 2020.

[13] Y. Q. Liu and G. L. Chang, "Research on influencing factors of knowledge sharing among mobile reading users in university libraries," Researches in Library Science, vol. 18, pp. 54-62, 2018.

[14] M. Li and Q. Cheng, "Research on influencing factors of mobile digital reading payment for college students based on consumer behavior theory," Researches in Library Science, vol. 13, pp. 55-61+96, 2018.

[15] L. C. Liu and X. Li, "Social reading users' exit, voice, loyalty and neglect behaviors from the perspective of psychological contract," The Journal of the Library Science in China, vol. 44, no. 4, pp. 89-108, 2018.

[16] Y. Wang, J. J. Shen, and Z. Jiang, "Research on mobile reading literacy in the context of algorithm recommendation in the mobile internet era," New Century Library, vol. 6, pp. 24-29, 2020. 
[17] R. J. Bian, "An empirical study on the influencing factors of customer satisfaction in mobile reading," Master Degree Thesis, Nanjing University of Posts and Telecommunications, Nanjing, China, 2018.

[18] W. J. Zhao, Y. Liu, and C. L. Li, "Research on classification of mobile reading service demand and satisfaction improvement in university libraries," Library and Information Service, vol. 63, no. 24, pp. 86-96, 2019.

[19] W. J. Zhao, J. Ren, C. L. Li, and Y. Liu, "Research on evolution and simulation of mobile reading service satisfaction group in university library," Research on Library Science, vol. 14, pp. $32-42+83,2020$.

[20] B. Y. Wang and Z. H. Deng, "Research on the quality measurement for mobile reading service: based on ANP-Fuzzy comprehensive evaluation method," Library Tribune, vol. 38, no. 2, pp. 71-80, 2018.

[21] Y. Huang and Z. Fan, "Developing a measurement scale for the usability of mobile reading application," Library Tribune, vol. 38, no. 2, pp. 119-125, 2018.

[22] X. W. Wang and M. M. Ren, "Research on the influence factors of mobile reading tools on user experience," Journal of Modern Information, vol. 39, no. 2, pp. 73-84, 2019.

[23] J. J. Li, F. Y. Ye, and Y. Y. Cao, "User experience dynamic behavior model and empirical study of mobile digital reading," Journal of Modern Information, vol. 39, no. 3, pp. 24-34+149, 2019.

[24] Z. Q. Li and J. H. Jiao, "Analysis of influence factors for customer satisfaction in WeChat based on DEMATEL," Journal of Modern Information, vol. 38, no. 12, pp. 114-119, 2018.

[25] P. C. Xu, Q. Bi, M. X. Ding, and J. Li, "Identification and analysis of DEMATEL-based of knowledge discovery system interaction quality influencing factors," Information and Documentation Services, vol. 29, no. 4, pp. 24-31, 2018.

[26] Y. Dang and Y. S. Yang, "Analysis on the influencing factors of the development of agricultural think tanks based on the DEMATEL method," Journal of Modern Information, vol. 39, no. 11, pp. 113-120+136, 2019.

[27] Z. Y. Jiang, Z. H. Xie, J. Chu, and K. Zou, "Identification of influence factors for public information service quality of smart cities from the perspective of information ecology," Information Science, vol. 38, no. 3, pp. 42-48+53, 2020.

[28] Y. Kang, "Identification of key factors affecting We chat public reading promotion in university libraries," Information Science, vol. 38, no. 6, pp. 139-144, 2020.

[29] M. J. Du, "Identification of supporting factors and policy inspiration on provincial capital population attraction," Regional Economic Review, vol. 9, no. 1, pp. 64-78, 2021.

[30] X. Y. Tang, J. Ma, and M. J. Wu, "Identification of network structure characteristics and influencing factors of traditional industrial clusters: based on complex social network analysis," Technology Economics, vol. 39, no. 12, pp. 147-154, 2020.

[31] G. Su, W. J. Wang, J. X. Guo, and H. B. Wang, "Research on the measurement of innovation factor structure and the identification of key influencing factors in modern economic system," Scientific Management Research, vol. 38, no. 6, pp. 18-25, 2020.

[32] A. Trivedi, S. K. Jakhar, and D. Sinha, "Analyzing barriers to inland waterways as a sustainable transportation mode in India: a dematel-ISM based approach," Journal of Cleaner Production, vol. 295, p. 126301, 2021.

[33] Y. Y. Ding and Z. Li, "Identification of constraints of innovative entrepreneurship education for college students based on grey-DEMATEL," Hubei Social Sciences, vol. 34, no. 1, pp. 151-157, 2020.

[34] X. D. Ji, Y. Xue, and C. Y. Xue, "Analysis on risk factors of crowd financing in equity-based agriculture from the perspective of investors: based on AHP-DEMATEL model," Modernization of Management, vol. 40, no. 1, pp. 105-109, 2020.

[35] Q. Zhang and C. H. Zhu, "Research of influence factors for China's municipalities entrepreneurial environment based on BP-DEMATEL," Industrial Technology \& Economy, vol. 37, no. 10 , pp. 67-74, 2018.

[36] B. W. Quesenbery, "Balancing the 5Es: usability," Cutter IT Journal, vol. 17, no. 2, pp. 4-11, 2004.

[37] P. Morville, "User experience design," 2004, http:// semanticstudios.com/publications/semantics/000029.php.

[38] D. Vyas and V. D. V. Gerrit, "APEC: a framework for designing experience," 2006, http://www.infosci.cornell.edu/ place/15_DVyas2005.pdf.

[39] A. K. Sangaiah, O. W. Samuel, X. Li, M. Abdel-Basset, and H. Wang, "Towards an efficient risk assessment in software projects-fuzzy reinforcement paradigm," Computers \& Electrical Engineering, vol. 71, pp. 833-846, 2018.

[40] A. K. Sangaiah, J. Gopal, A. Basu, and P. R. Subramaniam, “An integrated Fuzzy DEMATEL, TOPSIS, and ELECTRE approach for evaluating knowledge transfer effectiveness with reference to GSD project outcome," Neural Computing and Applications, vol. 28, no. 1, pp. 111-123, 2017.

[41] A. K. Sangaiah, P. R. Subramaniam, and X. Zheng, "A combined Fuzzy DEMATEL and fuzzy TOPSIS approach for evaluating GSD project outcome factors," Neural Computing and Applications, vol. 26, no. 5, pp. 1025-1040, 2015.

[42] N. Jamali, A. Sadegheih, M. M. Lotfi, L. C. Wood, and M. J. Ebadi, "Estimating the depth of anesthesia during the induction by a novel adaptive neuro-fuzzy inference system: a case study," Neural Processing Letters, vol. 53, no. 1, pp. 131-175, 2021.

[43] H. Jafari, M. T. Malinowski, and M. J. Ebadi, "Fuzzy stochastic differential equations driven by fractional Brownian motion," Advances in Difference Equations, vol. 2021, no. 1, p. 16, 2021.

[44] H. Jafari and M. J. Ebadi, "Malliavin calculus in statistical inference: cramer -rao lower bound for fuzzy random variables," Journal of Decisions \& Operations Research, vol. 5, no. 2, pp. 124-132, 2020.

[45] M. Rostami, K. Berahmand, and S. Forouzandeh, "A novel method of constrained feature selection by the measurement of pairwise constraints uncertainty," Journal of Big Data, vol. 7 , no. 1 , p. 83, 2020.

[46] R. J. Li, "Fuzzy method in group decision making," Computers and Mathematics with Applications, vol. 38, no. 1, pp. 91-101, 1999. 